\title{
Probing The Structure of Space-Time with Cosmic Rays
}

\author{
Roberto Aloisio ${ }^{1,4}$, Pasquale Blasi ${ }^{2}$, Piera L. Ghia ${ }^{3,5}$, Aurelio F. Grillo ${ }^{4}$ \\ ${ }^{1}$ Dipartimento di Fisica, Università di L'Aquila, Via Vetoio, 67100 Coppito (L'Aquila) - Italy \\ ${ }^{2}$ NASA/Fermilab Astrophysics Group, Fermi National Accelerator Laboratory, Box 500, Batavia,
}

\author{
IL 60510-0500 \\ ${ }^{3}$ Istituto di Cosmo-Geofisica del CNR, Corso Fiume 4, 10133, Torino, Italy \\ ${ }^{4}$ Laboratori Nazionali del Gran Sasso, INFN, SS. 17bis, Assergi (L'Aquila) - Italy \\ ${ }^{5}$ Istituto Nazionale di Fisica Nucleare, Via P. Giuria 1, 10125, Torino, Italy
}

\begin{abstract}
The study of the interactions of Cosmic Rays (CR's) with universal diffuse background radiation can provide very stringent tests of the validity of Special Relativity. The interactions we consider are the ones characterized by well defined energy thresholds whose energy position can be predicted on the basis of special relativity. We argue that the experimental confirmation of the existence of these thresholds can in principle put very stringent limits on the scale where special relativity and/or continuity of space-time may possibly break down.
\end{abstract}

\section{INTRODUCTION}

The study of interactions of high energy Cosmic Rays (nucleons and gamma rays) can provide severe tests of the validity of Special Relativity (SR). Indeed, SR is at the very base of our theories for the description of the Universe, and its validity is generally not questioned. However, several attempts have been made to put under scrutiny the postulates that SR relies upon, like the constancy of the velocity of light, and these studies have contributed accurate limits on possible violations (see e.g. [1] and references therein). 
Although it is very well proven that SR and the underlying Lorentz invariance (LI) provide a suitable framework for our low energy effective theories, it is not clear whether more ambitious theories, aiming to a global description of our World, including gravity, are or need to be Lorentz invariant. Theoretically, the need for a unified theory of gravity has led to several models, some of which automatically imply the breaking of LI. This is qualitatively understandable since the very concept of continuous space-time is likely to be profoundly changed by quantum gravitational effects [2]. In this prospective we think it is worth keeping an open mind with respect to the validity of what we define as fundamental theories, and always put them under experimental scrutiny.

This attitude appears even more justified at the present time: observationally, high energy astrophysics is providing a range of opportunities to probe energies (i.e. Lorentz factors, or speeds) much larger than the ones ever obtained, or obtainable in the future, in accelerator experiments. In particular we concentrate our attention on the interactions of cosmic gamma rays and nucleons with some type of universal photon background, i.e. the cosmic microwave (CMB), the far infrared (FIRB), and the radio background. These are the interactions responsible for gamma ray absorption from distant sources (through production of $e^{+} e^{-}$) [3] 5], that should appear as a cutoff in the gamma ray spectrum, and of the well known Greisen-Zatsepin-Kuzmin (GZK) cutoff [6,7] due to the photopion production in collisions of ultra high energy cosmic rays (UHECRs) off the CMB photons. All these processes have the same general structure: in a Lorentz invariant picture, these reactions would be examples of very low energy processes in the center of momentum, that appear boosted to very large Lorentz factors in the laboratory frame. Testing the presence of the thresholds for these processes is therefore a test of SR up to the large Lorentz factors in the boost.

Our approach is entirely phenomenological, and reasonably model independent. We do not propose, if not as examples, any specific model. We show that when a very general 
form of LI violation 1 is explicitly allowed in the dispersion relation between energy and momentum of particles, the threshold momenta for some of these reactions may drastically change or even become unphysical (i.e. the process does not occur), unless the LI violation is introduced at a length scale much smaller than the Planck scale. A similar approach, although more specific, was proposed in [8] (see Sect. 3).

The aim of the present paper is to discuss the possibility that future CR experiments could test Special Relativity and/or the structure of space-time. In our opinion, the (lack of) knowledge of the sources of the CR's under consideration does not allow us to support the idea [1,911] that the present experimental situation gives evidence for violation of LI or of continuity of space-time. Rather we stress that a verification of the existence of the quoted thresholds would entail a lower limit on the mass scale of effective LI violations.

The paper is planned as follows: in section 2 we discuss our parametrization of the LI violations, and discuss briefly some models in which these parametrizations hold; in section 3 we apply our calculations to the case of pair production and photopion production in high energy cosmic ray interactions. We conclude in section 4.

\section{BREAKING OF LORENTZ INVARIANCE}

Lorentz invariance implies that the modulus of any four-vector is unchanged when changing reference frame; for instance for the four-momentum of a particle we have (we always

\footnotetext{
${ }^{1}$ Clearly, breaking LI can have several implications, as for instance the existence of a preferred reference frame, which is the one in which all the calculations need to be carried out, since breaking LI also invalidates the transformations that allow us to change reference frame. This reference frame could be identified with the one comoving with the expansion of the universe (no peculiar motion), in which the microwave background is completely isotropic. In fact, there is only one frame with this property, being all other frames experiencing the dipole anisotropy, and therefore distinguishable.
} 
put $c=\hbar=1)$

$$
P_{\mu} P^{\mu}=E^{2}-p^{2}=\text { const }=m^{2}
$$

$m$ being the invariant particle mass and $p=|\vec{p}|$.

Violations of Lorentz invariance will therefore affect in general the dispersion relation above. Without referring to any specific model (later we will discuss explicit implementations), we write a modified dispersion relation obeying the following postulates:

1. Violations are a high energy effect, i.e. they vanish at small momenta.

2. Violations are universal, i.e. do not depend on the particle type, if not (possibly) through the particle mass.

3. Rotation invariance remains exact.

Clearly requirements 2 and 3 may be relaxed and in fact there are examples in this sense [1, 12]. We write then the modified dispersion relation as follows:

$$
E^{2}-p^{2}-m^{2}=p^{2} f\left(\frac{p}{M}\right)+m^{2} g\left(\frac{p}{M}\right)
$$

where the mass $M$ parametrizes the violation of Lorentz invariance (or an essential discreteness of space-time, as for instance suggested by some models of quantum gravity (e.g. [2] and [13,14])). Even in the framework defined above this is not the most general violation term. However, in the regime we are interested in, $m \ll p \ll M$, the left hand side of eq. (11) is small compared to $p^{2}$ and $E^{2}$ so that the other possible terms we can write (containing for instance $E$ ) differ from those in eq. (11) by higher order corrections \&.

Since $p / M \ll 1$ the functions $f$ and $g$ can be Taylor-expanded to give:

$$
E^{2}-p^{2}-m^{2}=p^{2}\left(f(0)+f^{\prime}(0) \frac{p}{M}+f^{\prime \prime}(0) \frac{p^{2}}{M^{2}}+\ldots\right)+
$$

\footnotetext{
${ }^{2}$ Also, terms proportional to $m p$ can be added. They however do not modify the general framework so we will not include them for sake of clarity.
} 


$$
m^{2}\left(g(0)+g^{\prime}(0) \frac{p}{M}+g^{\prime \prime}(0) \frac{p^{2}}{M^{2}}+\ldots\right)
$$

In the limit $M \rightarrow \infty$ one must recover the Lorentz invariant dispersion relation, so $f(0)=$ $g(0)=0$; moreover the linear term might be absent, as we will see later; if it is present, the quadratic term is negligible at the momenta we consider. The coefficients in front of the first and second derivatives are left unconstrained. However, if $\left|f^{\prime}(0)\right|,\left|g^{\prime}(0)\right| \gg 1$ or $\left|f^{\prime \prime}(0)\right|,\left|g^{\prime \prime}(0)\right| \gg 1$, then the functions $f$ and $g$ would be strongly varying and possibly oscillating, which would be unphysical. Moreover, in cases where the calculations of the LI breaking can be carried out explicitly (see section $2 \mathrm{~A}$ ), these coefficients turn out to be of order unity (the same motivation justifies the assumption that these coefficients are not much smaller than unity). Therefore we decided to adopt here a phenomenological approach and assume that possible small deviations from unity are embedded in the mass scale $M$. The validity of this approach will be checked a posteriori.

We are thus led to the following classification of Lorentz non-invariant dispersion relations:

$$
\begin{gathered}
I_{ \pm}: \quad E^{2}-p^{2} \approx m^{2} \pm \frac{p^{3}}{M} \quad\left( \pm \frac{m^{2} p}{M}\right) \\
I I_{ \pm}: \quad E^{2}-p^{2} \approx m^{2} \pm \frac{p^{4}}{M^{2}} \quad\left( \pm \frac{m^{2} p^{2}}{M^{2}}\right)
\end{gathered}
$$

where $I(I I)$ stands for first (second) order modification and the terms in parenthesis come from the expansion of $g$.

We can define a critical momentum $p_{c}$ where the correction, for massive particles, equals $m^{2}$, which is the momentum for which we expect that deviations from normal relativistic kinematics become relevant. We have $p_{c} \approx 2 \times 10^{15} \mathrm{eV}\left(\approx 10^{13} \mathrm{eV}\right)$ for protons (electrons) in the case $I_{ \pm}$and $p_{c} \approx 3 \times 10^{18} \mathrm{eV}\left(\approx 10^{17} \mathrm{eV}\right)$ for protons (electrons) in the case $I I_{ \pm}$. In the cases in parenthesis in eqs. (3) and (4) we have always $p_{c} \approx M$, so these modifications do not lead to observable consequences at the energies we are interested in; we will therefore put $g\left(\frac{p}{M}\right)=0$ in the following. We will come back to this point when we will describe specific 
examples of Lorentz violating theories [8]. It is clear that the values of $p_{c}$ given above are calculated in a specific frame; in fact, generally, violations of LI imply the existence of a privileged frame. We choose this frame to be the one comoving with the expansion of the universe (we name it "universal frame"), and we argue that this choice is in fact not arbitrary: this is the only possible frame where the microwave background is isotropic (the same holds for the other backgrounds, provided the sources are homogeneously and isotropically distributed). Moreover, neglecting the proper motion of the Earth, this is the reference frame in which we live and measure the thresholds for physical processes. On a more practical ground, it is worth noticing that giving up LI, the Lorentz transformations do not correctly give the transformations laws of energy and momentum between different frames although in principle it is possible to write modified transformations of energy and momentum (see for instance [15]) which reproduce, at least at the perturbative level chosen, the dispersion relations above. In this case, however, there is much more arbitrariness than in modifying the dispersion relation and we do not pursue this approach in this paper. It is worth noting however that the Lorentz Transformations are derived in a LI theory by the requirement of the invariance of a fundamental interval, so in a sense a modification of the dispersion relation is more fundamental.

There is a further, important point to discuss before computing particle production thresholds. In fact, when one gives up relativistic invariance, energy-momentum conservation is not anymore guaranteed, and relativistic quantum field theory may fail, so there is no guidance in deriving cross sections. However, our point of view here is to derive the consequences of experimental verification of the existence of the particle thresholds, so we will assume in the following exact energy-momentum conservation and relativistic dynamics in the preferential frame. 


\section{A. Models}

In the following we will briefly discuss some models in which modified dispersion relations are actually obtained. It has been argued on very general grounds that quantum gravity effects do modify particle propagation at scales close to the Planck scale. In particular,

modified uncertainty relations and existence of a minimum proper length (see e.g. 16] and references therein) (implying modifications of Lorentz transformations), and light-cone fluctuations (see e.g. [17] and references therein) are fairly general implications of quantum gravity and modify the dispersion relations.

An approach where a fundamental mass/momentum scale $M$ is introduced to parametrize the deviations from LI can be pursued by writing the commutators between (space) boosts and translation generators of the Poincare' algebra in a modified form [18], leading to

$$
\begin{array}{ll}
\text { - } I I_{+} & m^{2}=M^{2} \sin ^{2}\left(\frac{E}{M}\right)-p^{2} \sim E^{2}-p^{2}-\frac{E^{4}}{12 M^{2}} \\
\text { - } I I_{-} & m^{2}=M^{2} \sinh ^{2}\left(\frac{E}{M}\right)-p^{2} \sim E^{2}-p^{2}+\frac{E^{4}}{3 M^{2}}
\end{array}
$$

A theory with both exact conservation of energy and momentum and LI violations can be obtained if it is possible to construct a local theory which is symmetric under the modified Poincaré group 19

Models giving the $I_{ \pm}$dispersion relations can be derived from some quantum gravity approach. The basic idea is that quantum fluctuations of gravity cause, at a scale of Plank mass, the vacuum to behave like a stochastic medium and this introduces non-zero, energy dependent non-diagonal terms in the metric [13,

$$
\begin{array}{lll}
\text { - } I_{+}: & g_{\mu \nu} \rightarrow g_{\mu \nu}+h_{0 i} & h_{0 i}=U_{i} \quad \vec{U}=-\frac{E}{M} \hat{p} \\
\text { - } I_{-}: & g_{\mu \nu} \rightarrow g_{\mu \nu}+h_{0 i} & h_{0 i}=U_{i} \quad \vec{U}=\frac{E}{M} \hat{p}
\end{array}
$$

with consequent modifications of the dispersion relations. Similar modifications may hold in brane models with large (or even infinite) extra dimensions and $T e V$ scale quantum gravity [20], where in some cases Poincare' invariance is broken explicitely 21. 
In the models presented above, quantum gravity effects are described by fluctuations around a flat background metric. An entirely different approach is followed in the loop approach to quantum gravity (see e.g [14] and references therein) in which the geometry itself emerges non perturbatively. It has been shown that this leads to an essential discretization of space, and to modifications of the dispersion relations which fall in the class $I_{ \pm}$if parity is broken; the violation might be milder (or absent) in parity conserving models.

We finish quoting a pioneering approach by Kirshnitz and Chechin [8], stimulated by the appearance of the papers by Kuzmin and Zatsepin [7], and Greisen [6]. It is a classical approach, in which the free lagrangian of a (classical) point particle is modified. The theory is defined replacing the pseudo-Euclidean space-time of SR with a Finslerian space [22]. In this approach the dispersion relation becomes

$$
m^{2}=\left[1+\bar{g}\left(\alpha \frac{p^{2}}{E^{2}}\right)\right]\left(E^{2}-p^{2}\right)
$$

where $m$ is the particle mass, and $\bar{g}$ is a homogeneous function of the dimensionless

parameter $\alpha$ that parametrizes the LI violations, $\alpha=\frac{m^{2}}{M^{2}}$ in terms of the scale M. This gives rise to a mild violation, disappearing for massless particles. With an appropriate choice of the function $\bar{g}$, this gives rise to the terms in parenthesis in eqs. (3) and (4).

\section{THRESHOLD CALCULATIONS WITH MODIFIED DISPERSION RELATIONS}

In this section we describe the calculation of the kinematic thresholds for some processes in the framework of the modified dispersion relations between energy and momentum introduced in the previous section. We choose two processes which are of astrophysical relevance and that will be accessible to next generation cosmic ray experiments: pair production in photon-photon scattering and photopion production in nucleon-gamma scattering. The first process is responsible for the absorption of high energy gamma rays from distant sources, while the second is responsible for the well known (and currently unobserved) GZK cutoff. 


\section{A. $e^{+} e^{-}$production in photon-photon interactions}

The process under investigation is $\gamma \gamma \rightarrow e^{+} e^{-}$. The energy of the background photons

is taken as $\omega=|k|$ (where $k$ is the photon momentum) since at the typical background momenta (FIRB: $\omega \approx 0.01 \mathrm{eV}, \mathrm{CMB}: \approx 6 \times 10^{-4} \mathrm{eV}$, and radio: $\approx 4 \times 10^{-9} \mathrm{eV}$ for the peak of the corresponding radiance distributions) the corrections are entirely negligible. We compute the threshold, assuming that the CR particle and the background photon collide head-on, and the final particles are collinear. This is indeed not an arbitrary configuration, but the one that provides the minimum energy for which the process can occur in the universal frame.

Rotational invariance then implies that in this reaction the momenta of final particles are equal, and that particles move in the direction of the primary, so that the problem is one-dimensional; the energy of the background photon is assumed to be equal to the average for the photon background in consideration.

Let then $(E, p)$ be the energy and (modulus of) the momentum of the incident photon. Writing the relations of conservation of energy and momentum in the laboratory frame, and using the modified dispersion relation between energy and momentum, after some trivial algebra, and neglecting sub-leading terms in the range of momenta we are considering, we get the following general equations for the threshold:

$$
\begin{gathered}
I_{ \pm}: \quad \pm \alpha_{I} x^{3}+x-1=0, \\
I I_{ \pm}: \quad \pm \alpha_{I I} x^{4}+x-1=0,
\end{gathered}
$$

where $x=p_{t h} / p_{0}, p_{0}=m^{2} / \omega$ is the threshold for $M \rightarrow \infty$

(i.e. the usual threshold, $\approx 3 \times 10^{13} \mathrm{eV}$ for the FIRB as background, $\approx 5 \times 10^{14} \mathrm{eV}$ for the $\mathrm{CMB}$, and finally $\approx 6 \times 10^{19}$ for the radio background), $m$ the electron mass, and:

$$
\alpha_{I}=\frac{p_{0}^{3}}{8 m^{2} M} ; \quad \alpha_{I I}=\frac{3 p_{0}^{4}}{16 m^{2} M^{2}} .
$$


The modified thresholds are the positive real solutions of the equations above, and in particular, if more than one positive solution is present, the one which goes to $x=1$ as

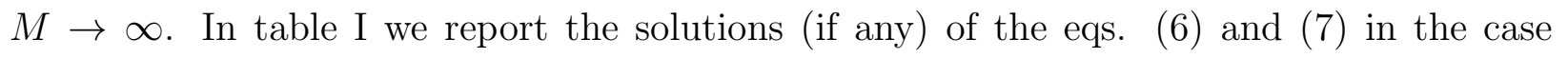
$M=M_{P}$, reasonable in quantum gravity models, for the infrared, microwave, and radio background.

The general feature of these solutions is that a positive modification of the dispersion relation tends to move the thresholds towards lower momentum values; negative modifications tend to lead to complex solutions, meaning that the kinematics of the process becomes forbidden, i.e. the threshold disappears. Case II is less predictive, as it is obvious being the modification of second order in the (small) quantity $p / M$. Clearly a more detailed calculation, accounting for the integration over different scattering angles and over the whole spectrum of the photon background, could give slightly different results, not changing however our basic findings.

To assess the role of CR experiments to detect violations of LI we now assume that in present or future experiments it will be possible to isolate the effect of pair production on different photon backgrounds, by measuring the respective thresholds, with an experimental uncertainty on the measurement of the threshold energy of $\approx 100 \%$. This is a conservative (and probably pessimistic) approach, unless possibly for the radio case. We therefore derive a lower limit on $M\left(\neq M_{P}\right)$, which we report in table $\llbracket$.

Note that all these limits are more stringent than the few limits on $M$ already obtained (see for instance [23]). Exceptions to this statement come from the cases $I I_{ \pm}$for the IR and microwave backgrounds, where the limits on $M$ are appreciably smaller that $M_{P}$, leaving more room for non Lorentz invariant theories.

We now discuss the present situation of VHE/UHE $\gamma$-ray astronomy in view of the possibility of testing LI. In doing this it is important to keep in mind the quite different observational situation existing for the CMB versus the IR and Radio backgrounds: while the former is determined with very high accuracy, the IR and Radio backgrounds are very poorly known and difficult to access through observational investigation, mainly due to the 
emission and/or absorption processes in our Galaxy.

Observationally, the infrared gamma-ray cutoff seems to be the one more easily approachable. Indeed signs of a cutoff in the $\mathrm{TeV}$ spectrum of a few blazars seem to be already present ( [24-28]) and helped to impose some constraints on the extragalactic far-infrared background (FIRB). Unfortunately, as it is clear from table $\mathbb{E}$, the case of interaction with the FIRB is not very predictive, in the sense that the threshold is not appreciably changed with respect to the case of a Lorentz-invariant theory, for most of the possible parametrizations of LI breaking (using $M=M_{P}$ ). The case $I_{-}$is an exception: in this case there is no acceptable solution of the equations that define the threshold, which means that the process is not possible at all. In other words, in this case no cutoff should be seen in astrophysical observations because photons can come unattenuated from all distances. Viceversa, if a cutoff is observed, then a lower limit on $M$ can be imposed.

As far as the photon absorption on the CMB radiation is concerned, the experimental situation is more uncertain, due to the higher energy of the $\gamma$-rays involved (although there might be suggestion of attenuation due to CMB, see e.g. 22, 30, This is theoretically more restrictive: in the scenario $I_{+}$the threshold is moved to smaller values. For $M=M_{P}$, photons with energy as small as $\sim 25 \mathrm{TeV}$ from a distant source should be absorbed. In order for the threshold to be unchanged, the scale $M$ must exceed $\sim 800 M_{P}$. In the scenario $I_{-}$ the process becomes not allowed, while in the other cases the threshold remains unchanged.

The case of scattering off the radio background is the most interesting. We predict, for $M=M_{P}$, either no threshold (cases $I_{-}$and $I I_{-}$) or thresholds which are much smaller that the canonical ones. This could be of great importance for top-down models of UHECRs, where gamma rays are supposed to have an important role in the composition (e.g. see [31). In particular the proton/gamma ratio is determined by the interaction of the gamma rays with the radio background at frequencies smaller than a few MHz. Unfortunately, as mentioned above, the radio background at these frequencies is extremely uncertain, and not accessible to any direct measurement, due to the strong free-free absorption in the disc and halo of our own Galaxy. 


\section{B. Pion photoproduction in UHECR interactions: the GZK cutoff}

This case is slightly more involved since the masses of the final particles are different, so that even for a rotation invariant modification, final momenta are not in the same ratio as the masses. However we checked that assuming the ratio of final momenta as in the Lorentz invariant theory, we introduce only higher order corrections. Using this prescription we obtain the following two equations for the threshold, with the same symbols used in the previous section:

$$
\begin{gathered}
I_{ \pm}: \quad \pm \alpha_{I} x^{3}+x-1=0 \\
I I_{ \pm}: \quad \pm \alpha_{I I} x^{4}+x-1=0
\end{gathered}
$$

where $x=\frac{p_{t h}}{p_{0}}$ and $p_{0}=\frac{m_{\pi}^{2}+2 m_{\pi} m_{p}}{4 \omega}$ is the conventional threshold (for $M \rightarrow \infty$ ), $m_{p}$ is the proton (neutron) mass, and $m_{\pi}$ is the pion mass. The coefficients $\alpha_{I, I I}$ are defined as follows:

$$
\begin{gathered}
\alpha_{I}=\frac{2 p_{0}^{3}}{\left(m_{\pi}^{2}+2 m_{\pi} m_{p}\right) M} \frac{m_{\pi} m_{p}}{\left(m_{\pi}+m_{p}\right)^{2}} \\
\alpha_{I I}=\frac{3 p_{0}^{4}}{\left(m_{\pi}^{2}+2 m_{\pi} m_{p}\right) M^{2}} \frac{m_{\pi} m_{p}}{\left(m_{\pi}+m_{p}\right)^{2}} .
\end{gathered}
$$

Fixing $M=M_{P}$ we have the solutions reported in table [II].

The general trend of the solutions is the same as in the case of pair production. Here however we can easily see that the consequences are even more evident: in all cases either the threshold disappears and the process becomes forbidden (cases $I_{-}$and $I I_{-}$), or the threshold is appreciably lowered (cases $I_{+}$and $I I_{+}$). For $I_{-}$or $I I_{-}$parametrizations, the GZK cutoff is completely washed out and particles (nucleons) should be able to reach us from any distance. In the other cases the threshold falls in a region that is easy to discard even on the basis of the present data, unless the scale of breaking of LI is $\sim 3 \times 10^{13}(\sim 500)$ times larger than the Planck mass for the case $I_{+}\left(I I_{+}\right)$. 
As in the previous section, if one assumes that the apparent absence of the GZK cut-off is an incidental fact, possibly due to our ignorance of the sources of CR's at these energies, and that CR experiments may find some remnant of the cut-off, we can derive limits on the parameters of LI violations. These limits are reported in table III, again assuming a $100 \%$ error on the location of the cut-off. From the values reported it is clear that UHECR experiments soon to be available will provide a powerful tool to explore the very-small-scale structure of space-time.

\section{CONCLUSIONS}

Special Relativity is at the base of our understanding of the physical world. However any physical concept should always be put under stringent experimental verification. This is particularly true in the case of SR, specially in connection with the quest for a fundamental theory of Nature, which includes gravity. Any such theory will imply a full knowledge of what the vacuum really is at the level where quantum fluctuations build the space-time,

possibly modelling it with a non-trivial geometry. Some first attempts to reach this goal seem to suggest that Lorentz invariance, one of the building blocks of our current low energy theories, could be broken at extremely high energy.

Lacking a true theory of quantum gravity, the most we can do at present is to adopt a phenomenological approach and ask ourselves whether there is any probe that can be used to check the validity of SR at very high energy. This is precisely the approach adopted in this paper, where high energy cosmic rays (nucleons and gamma rays) have been used as probes. We found that the thresholds for pair production and photopion production off some universal photon backgrounds, which are experimentally accessible or will be in the next generation of cosmic ray experiments, are often profoundly affected by the possibility of breaking LI at supra-Planck scales. As a consequence, any quantum gravity theory in which LI is a casualty must face the cosmic ray bound in order to be viable.

Our calculations were based on a perturbative but quite general modification of the 
dispersion relation between energy and momentum of a particle. This modification affects in a fundamental way the calculation of the thresholds for pair production and photopion production, making these processes forbidden in some cases, or lowering the thresholds to questionable values in others. Building on this approach we proposed a procedure to obtain very strong constraints on the energy (or length) scale at which a possible LI violation could occur.

This procedure relies upon the possibility that astrophysical observations will be able in the near future to find evidence for these processes, through cutoffs in the TeV spectra of distant blazars or/and through the discovery of possible consequences of the GZK cutoff in the spectrum of UHECRs or/and through studies of the composition of UHECRs. With a few exceptions, these limits are all higher than the Planck scale, which is an indication that breaking LI is not necessarily a safe ingredient of unified theories that require it.

We stress that our approach is purely kinematical and no dynamical effect is considered. Moreover, we assume perfect energy-momentum conservation in order to compute the thresholds. While these are clearly important issues for any calculation aiming to predict values for specific particle production thresholds, and consequently absorption cutoffs in CR spectra, our approach is rather the opposite: we want to discuss the consequences of a possible experimental verification of the presence of particle production thresholds. If a given absorption threshold is experimentally detected, discarding the possibility of miraculous compensations between relativity violations, non conservation of energy-momentum and non-relativistically invariant dynamics, we are forced to conclude that possible violations of LI are smaller than the sensitivity of the experiment, and, to make this statement quantitative, we choose to use a purely kinematical, energy-momentum conserving parametrization.

Although a very tempting possibility, we do not believe that the current experimental situation allows us to draw definitive statements : recent observations of Markarian 501 might suggest the presence of a cutoff in the $\mathrm{TeV}$ region, but the unaffected spectrum is not known well enough to exclude that the observed effect is the artifact of a cutoff in the production spectrum. Hopefully the situation will improve with the next generation gamma 
ray detectors, complemented by neutrino and X-ray detectors that could clarify the origin of the gamma ray emission. Concerning $\gamma$-rays absorption on CMB photons, experimental data are more scarce, and the situation is not likely to improve in the near future.

Pair production on the universal radio background becomes relevant for ultra-high-energy gamma rays, usually produced in top-down models of UHECRs [31]. The presence or absence of a threshold for this process strongly affects our predictions of the fluxes at the Earth: although very uncertain, the radio background should allow typical gamma ray pathlengths (in a Lorentz invariant world) of the order of $2-20 \mathrm{Mpc}$ [32]. If a violation of LI made the pair production kinematically forbidden, gamma rays could reach us from any distance and contribute an enormous flux of particles above the GZK cutoff.

As for UHECRs, although the (small) number of events 33, 34 above $\sim 5 \times 10^{19} \mathrm{eV}$ seems already incompatible with the presence of the GZK cutoff, the lack of knowledge of the sources does not allow any firm statement on the propagation of primaries in the Universe. The situation will improve dramatically in the near future, as new experiments like HiRes [35] and Auger [36] will collect data and reliable measurements on observables different from the energy spectrum (anisotropy, clustering) will be available with reasonable statistics.

Our conclusions can be summarized in the following points: 1) it is a 40-years old idea that the incompleteness of the present theories could rely upon our ignorance of the vacuum at very high energies. When we will have that knowledge we will probably have a quantum theory of gravity; 2) several attempts to quantize gravity have naturally led to the requirement of violations of the LI; 3) experiments on cosmic rays can represent the most easily approachable tool to probe the structure of space-time on the very small scales; 4) LI violations affect the thresholds for elementary processes relevant for cosmic ray astrophysics and can be observationally tested.

After the submission of this paper we have noticed some papers [37 which reach similar conclusions. 


\section{ACKNOWLEDGEMENTS}

We are grateful to V. Berezinsky and G. Di Carlo for very useful and stimulating discussions. The work of P.B. was funded by the DOE and the NASA grant NAG 5-7092 at Fermilab. 


\section{REFERENCES}

[1] S. Coleman, S.L. Glashow, Phys. Rev. D59, 116008 (1999).

[2] J.A. Wheeler, Annals of Physics 2, 604 (1957).

[3] A.I. Nikishov, Sov. Phys. - JETP 14, 393 (1962).

[4] P. Goldreich and P. Morrison, Sov. Phys. - JETP 18, 239 (1964).

[5] R.J. Gould and G.P. Schreder, Phys. Rev. Lett. 16, 252 (1966).

[6] K. Greisen, Phys. Rev. Lett. 16, 748 (1966).

[7] G.T. Zatsepin and V.A. Kuzmin, Pis'ma Zh. Ekps. Teor. Fiz. 4, 114 (1966) [JETP Lett. 4, 78 (1966)].

[8] D.A. Kirzhnits and V.A. Chechin, Sov. Jour. Nucl. Phys. 15, 585 (1971).

[9] L. Gonzalez-Mestres, Proc. 26th ICRC (Salt Lake City, USA), 1, 179 (1999).

[10] W. Kluzniak, Astropart. Phys. 11, 117 (1999).

[11] T. Kifune, Astrophys. J. Lett. 51821 (1999).

[12] G.Yu. Bogoslovsky and H.F. Goenner, preprint gr-qc/9904081.

[13] J. Ellis, N.E. Mavromatos and D.V. Nanopoulos, preprint hep-th/9909085.

[14] R. Gambini and J. Pullin, Phys. Rev. D59 124021 (1999).

[15] G. Yu. Bogoslovsky, Fortschr. Physik 42, 143 (1994).

[16] J.L. Garay, Int. J. Mod. Phys. A14 4079 (1999).

[17] L.H. Ford and Hongwei Yu, preprint gr-qc/9907037.

[18] J. Lukierski, preprint hep-th/9812063.

[19] P. Kosinski, J. Lukierski and P. Maslanka, preprint hep-th/9902037. 
[20] J. Ellis, N.E. Mavromatos and D.V. Nanopoulos, Phys. Rev. D61 027503 (2000).

[21] G. Dvali and M. Shifman, preprint hep-th/9904021, To appear in L.B. Okun Festschrift, Eds. V. Telegdi and K. Winter, to be published by North Olland.

[22] H. Rund, "The Differential Geometry on Finsler Spaces", Berlin 1959.

[23] S.D. Biller et al., Phys. Rev. Lett. 83, 2108 (1999).

[24] F. Krennrich et al., Astrophys. J. 511, 149 (1999).

[25] F.A. Aharonian et al., Astron. \& Astroph. 349, 11 (1999).

[26] V.V. Vassiliev, preprint astro-ph/9908088.

[27] F.W. Stecker, preprint astro-ph/9904416.

[28] P.S. Coppi and F.A. Aharonian, Astropart. Phys. 11, 35 (1999).

[29] M. Catanese et al., Astrophys. J. 469, 572 (1996).

[30] P.L. Ghia et al., Nucl. Phys. B (Proc. Suppl.) 70, 506 (1999).

[31] V.S. Berezinsky, P. Blasi and A. Vilenkin, Phys. Rev. D58, 103515 (1998).

[32] R.J. Protheroe and P.L. Biermann, Astropart. Phys. 6, 45 (1996); Erratum-ibid 7, 181 (1997).

[33] M. Takeda et al., Astrophys. J. 522, 225 (1999).

[34] T. Abu-Zayyad et al., Proc. 26th ICRC (Salt Lake City, USA), 3, 264 (1999).

[35] S.C. Corbato et al., Nucl. Phys. B (Proc. Suppl.) 28B, 36 (1992).

[36] J.W. Cronin, Nucl. Phys. B (Proc. Suppl.) 28B, 213 (1992).

[37] O. Bertolami, preprint gr-qc/0001097. 


\section{TABLES}

\begin{tabular}{lccc}
\hline \hline & Infrared & Microwave & Radio \\
\hline$I_{+}$ & $\approx 0.73$ & 0.06 & $5 \cdot 10^{-7}$ \\
$I_{-}$ & No solution & No solution & No solution \\
$I I_{+}$ & $\approx 1$ & $\approx 1$ & $2 \times 10^{-3}$ \\
$I I_{-}$ & $\approx 1$ & $\approx 1$ & No solution \\
\hline \hline
\end{tabular}

TABLE I. Values of $x$ that solve the equation for the threshold for pair production in the non-Lorentz invariant approach.

\begin{tabular}{lccc}
\hline \hline & Infrared & Microwave & Radio \\
\hline$I_{+}$ & $M \gtrsim 0.2 M_{P}$ & $M \gtrsim 800 M_{P}$ & $M \gtrsim 2.5 \times 10^{18} M_{P}$ \\
$I_{-}$ & $M \gtrsim 6 M_{P}$ & $M \gtrsim 3 \times 10^{4} M_{P}$ & $M \gtrsim 8 \times 10^{19} M_{P}$ \\
$I I_{+}$ & $\left(M \gtrsim 3 \times 10^{-8} M_{P}\right)$ & $\left(M \gtrsim 7 \times 10^{-6} M_{P}\right)$ & $M \gtrsim 10^{5} M_{P}$ \\
$I I_{-}$ & $\left(M \gtrsim 3 \times 10^{-7} M_{P}\right)$ & $\left(M \gtrsim 10^{-4} M_{P}\right)$ & $M \gtrsim 10^{6} M_{P}$ \\
\hline \hline
\end{tabular}

TABLE II. Limits on the scale $M$ where the LI is broken.

\begin{tabular}{lcc}
\hline \hline & $x$ & Limit \\
\hline$I_{+}$ & $2 \times 10^{-5}$ & $M \gtrsim 3 \times 10^{13} M_{P}$ \\
$I_{-}$ & No solution & $M \gtrsim 10^{15} M_{P}$ \\
$I I_{+}$ & 0.02 & $M \gtrsim 536 M_{P}$ \\
$I I_{-}$ & No solution & $M \gtrsim 6 \times 10^{3} M_{P}$ \\
\hline \hline
\end{tabular}

TABLE III. Values of $x$ that solve the equations for the threshold of photopion production on the microwave background (first column) and lower limit on the scale $M$ of breaking of LI (second column). 